\title{
A Comparative Study of Utilization of Single and Hybrid Data Mining Techniques in Heart Disease Diagnosis and Treatment Plan
}

\author{
Rajesh Jagtap \\ M.E.Scholar \\ Department of Computer Engg. \\ DPCOE,Wagholi,Pune
}

\begin{abstract}
In clinical medicine, data mining deals with learning models to predict patient's health. The models can be dedicated to support clinicians in diagnostic and monitoring tasks. Data mining methods are commonly applied in clinical contexts to analyze retrospective data, thus giving healthcare professionals the opportunity to exploit enormous amounts of data routinely collected during their day-by-day activity. Nowadays, clinicians can take advantage of data mining techniques to deal with the huge amount of research results obtained by molecular medicine such as genomic signatures or genetic which may allow transition from population-based to personalized medicine.The different classification and prediction models can be devoted to support medical practitioners in diagnosis and formation of treatment plans. There is need of powerful data analysis tool to extract useful knowledge from huge amount of data available in health care field. Last few years, heart disease is the major cause of death all over the world. In heart disease diagnosis and treatment, single data mining techniques are showing satisfactory level of accuracy. Nowadays, researchers are experimenting the deployment of hybrid data mining techniques showing great level of accuracy. In this paper,single data mining techniques like Naive base,Decision tree, Association rule, Neural network and Regression are studied and compared with hybrid data mining algorithm to achieve an efficient results in heart disease diagnosis and to formulate treatment plan.
\end{abstract}

\section{General Terms}

Healthcare, Diagnosis

\section{Keywords}

Hybrid, Data Mining, Genomic, Retrospective, Monitoring

\section{INTRODUCTION}

The heart is vital organ of human body which pumps blood through the body. Due to inefficient circulation of blood in body organs like brain, suffer and if heart stops working, death occurs within minutes. Human body operation is totally dependent on efficient working of the heart.Heart disease term refers to disease of heart

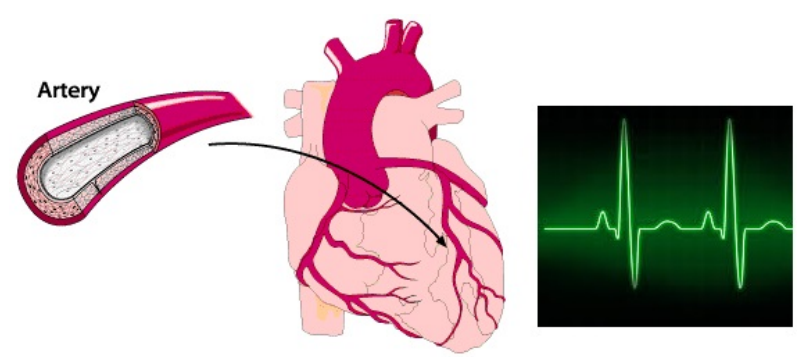

Fig. 1. Structure of Heart and ECG Diagnosis, ecgguru.com et al.[12]

and blood vessel system within it and includes the diverse diseases that affect the heart. Coronary heart disease, cardiovascular disease and Cardiomyopathy are some categories of heart diseases.Today, in the world, Heart disease is the major cause of deaths. The World Health Organization (WHO) has estimated that 13 million deaths occur worldwide, every year due to the cardiac diseases. WHO estimated by 2040 , almost 24.6 million people will suffer due to cardiac disease. [1]. The following are number of factors that increases the risk of Heart disease [2]:

(1) Hyper tension

(2) obesity

(3) Smoking

(4) Poor diet

(5) High blood pressure

(6) Physical inactivity

(7) High cholesterol

(8) Family history

The Diagnosis of patient is generally based on symptoms, signs, and physical examination and related to the classification of patients into disease classes or sub-classes on the basis of patient's data. This activity covers a broad spectrum of clinical cases, including triage at hospital emergency departments, i.e, prioritizing patients based on the severity of their condition.Practically all the doctors are diagnosing heart disease by knowledge and experience. The diagnosis of disease is a challenging and tedious task in medical field.Predicting cardiac disease from numerous 
factors or symptoms is a multi-layered issue which may lead to inappropriate presumptions and unpredictable effects. Nowadays, Healthcare industry generates large amounts of complex data about patients, hospitals resources, electronic patient records, disease diagnosis, medical devices etc. Only human intelligence alone is not sufficient for right diagnosis. A number of complications always arrive during diagnosis, such as less accurate results, time dependent performance, less experience, knowledge upgradation is difficult.

Data mining is a knowledge finding technique to examine data and summarize it into valuable information [2].The data mining techniques are utilized to pre-process the information from the patient's medical record and classify the attributes. The current research aims to predict the possibility of getting heart disease according to dataset of patient's medical record . In practice, Predictions and descriptions are primary goals of data mining [6]. Prediction in data mining comprises attributes or variables in the dataset to detect an unknown or future state values of other attributes [7]. Description highlight on discovering patterns that explains the data to be interpreted by humans.

\section{OBJECTIVES OF THE RESEARCH}

(1) To find out the accuracy of single data mining techniques and compare it with the accuracy of hybrid data mining techniques to diagnose the heart disease.

(2) To exploit the usefulness of hybridized data mining techniques in heart disease diagnosis and discover the suitable treatment for heart disease patients.

(3) To highlight the importance of computer algorithms in medical applications.

(4) To help medical practitioners to reduce the errors and complexity in diagnosis process and to improve the relationship between patient and medical practitioner.

\section{RELATED WORK}

Several studies have been done that have attention on diagnosis of heart disease. Researchers have applied different data mining techniques for diagnosis and achieved dissimilar probabilities for different methods.

\section{Data Mining Techniques used in Heart disease diagnosis:}

Mai Shouman, Tim Turner, Rob Stocker et al.[1] have shown the comparison of single and hybrid data mining techniques in the diagnosis of heart disease on the CHDD (Cleveland Heart Disease Dataset.The baseline accuracy got in diagnosis using single data mining technique is compared with baseline aacuracy got in treatment and The baseline accuracy got in diagnosis using hybrid data mining technique is compared with baseline accuracy got in treatment.These techniques shown different accuracies, where hybrid techniques were more accurate than single techniques accuracy.The best accuracy achieved using single data mining technique was $84.14 \%$ by naive bayes. However, the best accuracy achieved using hybrid data mining technique was $89.01 \%$ by neural network ensemble.Finally,they observed that hybrid data mining techniques are more accurate and enhanced the accuracy of heart disease diagnosis.

Supervised and Unsupervised learning method:

Mary K. Obenshain et al. [2] focused on data mining product-SAS Enterprise Miner,which often included in data mining application suites for specific application areas such as customer relationship
management(CRM), financial management.They have also highlighted the importance of supervised and unsupervised learning methods. Supervised learning methods deployed when values of variables (inputs) are used to make predictions about another variable (target) with known values. The supervised learning methods are used to make prediction about fraudulent claims using different attributes in healthcare organizations.In supervised methods, the models and attributes are known and are applied to the data to predict and find information.Unsupervised learning methods applicable almost in same situations, but are more regularly deployed on data for which a target with known values does not exist.

\section{Machine learning algorithms for data mining tasks:}

Rajkumar, A. and G.S. Reena et al. [3] proposed the utilization of Tanagra software used to compare the performance accuracy of data mining algorithms for diagnosis of heart disease dataset. The feature selection in the Tanagra software defined the attribute status of the data present in the heart disease.The authors have compared different supervised machine learning algorithms such as Naive Bayes, k-nn and Decision list. For data mining tasks, Tanagra was a proved to be successful tool which contains collection of machine learning algorithms. In their research, Naive Bayes algorithm shown the best compact time for processing dataset and better performance in accuracy prediction. The time required to run the data for result is faster when compared to other algorithms. It illustrated the enhanced performance according to input attribute. The attributes are entirely classified by this algorithm and it gave $52.33 \%$ of accurate result. According to the experimental results the classification accuracy is found to be better using Naive Bayes algorithm as compare to other algorithms. So, it was found that Naive Bayes algorithm plays a crucial role in shaping improved classification accuracy of a dataset.

\section{Verification of clinical data using SOAP:}

Razali and Ali et al. [4] surveyed the making of treatment plans for critical upper respiratory infection disease patients using a decision tree.Their study focused on outpatient and was based on data collected from various health centers throughout Malaysia. They have verified clinical data using SOAP (Subjective, Objective, Assessment and Plan) format approach as being practiced in medicine and were recorded electronically via Percuro Clinical Information System (Percuro). Cross-Industry Standard Process for Data Mining (CRISP-DM) model has been applied for the entire research. The data mining analysis is completed through decision trees technique with $\mathrm{C} 5$ algorithm. The scopes that have been set are patients complaint, age, gender, type of plan and detailed item given to patient. The suggested treatment model gave $94.73 \%$ accuracy through giving drugs to patients. The association rules and decision tree to treatment plans were shown satisfactory level of performance. They also found that the comparison of decision tree technique with other data mining techniques such as genetic algorithms, naive bayes, and neural network still needs further investigation.

\section{Utilization of decision tree:}

Kim et al.[6] evaluated the recent treatments for chronic heart failure using a decision tree and compared the results with those of large-scale clinical trials. They explored the procedures which recommended prescriptions of drugs to increase or decrease plasma level, spontaneous hypertension, fractional shortening and left ventricular end-diastolic diameter in the cardiovascular disease. However, they were unsuccessful to inspect exact data 
mining techniques to identify the suitable treatment for heart disease patients.

\section{Classification based data mining :}

Srinivas, K., B.K. Rani, and A. Govrdhan et al. [7] briefly examined the probable use of classification based data mining techniques such as Decision tree, Naive Bayes and Rule based Artificial Neural Network to huge volume of healthcare data. They have provided a well-organized approach for the extraction of significant patterns from the heart disease data warehouses for the efficient prediction of heart attack based on the calculated significant weightage, the frequent patterns having value superior than a predefined threshold were chosen for the valuable prediction of heart attack.For data preprocessing and effective decision making One Dependency Augmented Naive Bayes classifier (ODANB) and naive credal classifier 2 (NCC2) were utilized. That was an extension of naive Bayes to imprecise probabilities that aims at delivering robust classifications also when dealing with small or incomplete data sets.

\section{SINGLE DATA MINING TECHNIQUES}

In the healthcare field, data mining techniques have been utilized to help medical practitioner in the diagnosis of heart disease. Researchers are suggesting that applying data mining techniques in identifying effective treatments for patients can upgrade practitioners performance and helping to detect which data mining technique can provide more consistent accuracy.

\section{(1) Naive Bayes}

The Naive Bayes algorithm [13] is based on conditional probabilities and it is mostly suitable when the dimensionality of the inputs is very high where attributes are independent of each other. Naive Bayes model detects the characteristics of patients with heart disease. It shows the probability of each input attribute for the predictable state. It uses Bay's theorem. A conditional probability is the likelihood of some conclusion, $\mathrm{C}$, given some evidence/observation, $\mathrm{E}$, where a dependence relationship exists between $\mathrm{C}$ and $\mathrm{E}$.

This probability is denoted as $P(C \mid E)$ where

$$
P(C \mid E)=\frac{P(E \mid C) P(C)}{P(E)}
$$

Where,

$\mathrm{P}(\mathrm{C})=$ Prior probability of hypothesis $\mathrm{C}$

$\mathrm{P}(\mathrm{E})=$ Prior probability of training data $\mathrm{E}$

$P(C \mid E)=$ Prior probability of $\mathrm{C}$ given $\mathrm{E}$

$P(E \mid C)=$ Prior probability of $\mathrm{E}$ given $\mathrm{C}$

\section{(2) Neural Network}

A neural network $(\mathrm{NN})$ is a parallel, distributed information processing structure consisting of multiple numbers of processing elements called node, they are interconnected via unidirectional signal channels called connections. Each processing element has a single output connection that branches into many connections; each carries the same signal i.e. the processing element output signal.

The NN can be classified in two main groups according to the way they learn:

(a) Supervised learning

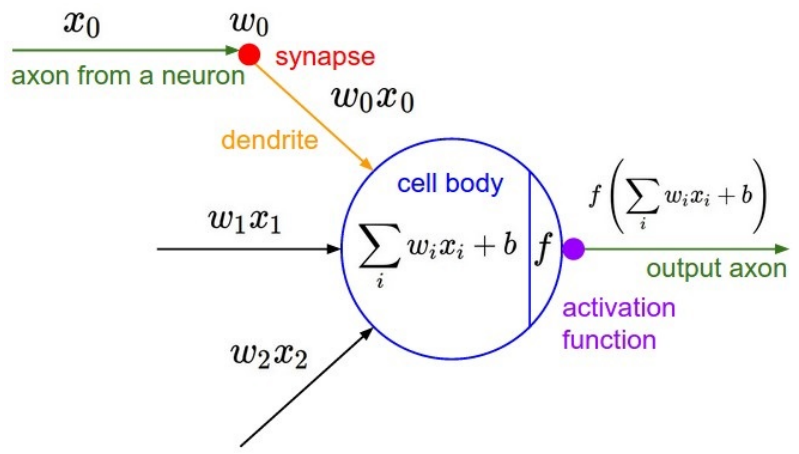

Fig. 2. Structure of Multi Layer Perceptron Neural Network,et al.[14]

It is a simple model, in which the networks compute a response to each input and then compare it with target value. If the computed response differs from target value, the weights of the network are adapted according to a learning rule.e.g. Multilayer Perceptron.

(b) Unsupervised learning

These networks learn by identifying special features in the problems they are exposed to. e.g.: Self-organizing feature maps.

\section{(3) Decision Tree}

Decision Tree techniques has shown useful accuracy in the diagnosis of heart disease.A decision tree is generated using C4.5 algorithm. It can be used for classification, which builds decision trees from a set of training data, using the concept of information entropy [11]. The training data is a set $\mathrm{T}=\mathrm{t} 1$, t 2 , t $3 \ldots$ of already classified samples. Each sample Si consists of a p-dimensional vector $(x 1, i, x 2, i, x 3, i, \ldots . . x p, i)$ where the $\mathrm{xj}$ represent attributes or features of the sample, as well as the class in which $\mathrm{Si}$ falls. At each node of the tree, C4.5 selects the attribute of the data that most efficiently splits its set of samples into subsets enriched in one class or the other. The splitting criterion is the normalized information gain (difference in entropy). The attribute with the maximum normalized information gain is selected to make the decision. The algorithm C4.5 then repeats on the smaller sublists.

The typical algorithm for building decision trees is:

(a) Verify all base cases

(b) For each attribute at

i. Calculate the normalized information gain ratio from splitting on $a t$

(c) Let at_best be the attribute with the maximum normalized Information gain

(d) Create a decision node that splits on at_best

(e) Repeat on the sublists obtained by splitting on at_best and add those nodes as children of node

(4) Linear Regression

Regression technique can be adapted for predication. Regression analysis can be used to model the relationship between one or more independent variables and dependent variables.It is a data mining function that predicts a number.A regression procedure begins with a data set in which the target values are known [10]. In the regression model relationships between predictors and target are summarized in a model, 


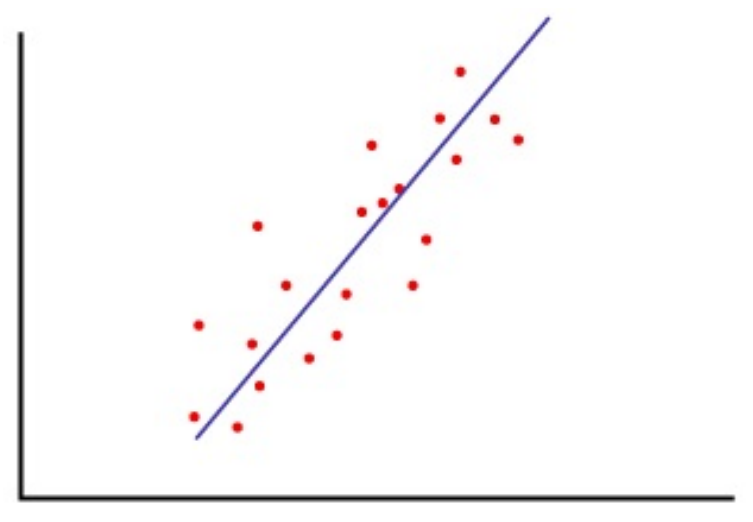

Fig. 3. Linear representation of data,et al.[15]

which can then be applied to a different data set in which the target values are unknown.A regression is a statistical analysis assessing the association between two variables. It is used to find the relationship between two variables.The relationship takes the form of an equation for a line that best indicates a series of data[15].For example, the line shown in the figure-3 is the best possible linear representation of the data.Each data point in the figure has an error associated with its distance from the regression line. The coefficients $\mathrm{a}$ and $\mathrm{b}$ in the regression equation adjust the angle and location of the regression line. For obtaining the regression equation,the adjustment of a and $b$ until the sum of the errors that are associated with all the points reaches its minimum.

\section{(5) Association Rule}

Association and correlation is usually to find frequent item set findings among large data sets.Association rule learning is a well-researched method for discovering interesting relations between variables in large databases [7]. It is intended to determine strong rules discovered in databases using different measures of interestingness. This type of finding helps businesses to make certain decisions, such as catalog design, cross marketing and customer shopping behavior analysis. Association Rule algorithms need to be able to generate rules with confidence values less than one. However the number of possible Association Rules for a given dataset is generally very large and a high proportion of the rules are usually of little (if any) value.

\section{- Mathematical Model of Association Rule}

The problem of association rule mining is defined as: Let $I=\{i 1, i 2, \ldots, i n\}$ be a set of $\mathrm{n}$ binary attributes called items. Let $D=\{t 1, t 2, \ldots, t n\}$ be a set of transactions called the database. Each transaction in $\mathrm{D}$ has unique transaction ID and contains a subset of the items in I . A rule is defined as an implication of the form $X \rightarrow Y$ where $X, Y \subseteq I$ and $X \cap Y=\phi$. The sets of items (for short item sets) $\mathrm{X}$ and $\mathrm{Y}$ and are called antecedent (left-hand-side or LHS) and consequent (right-hand-side or RHS) of the rule respectively.

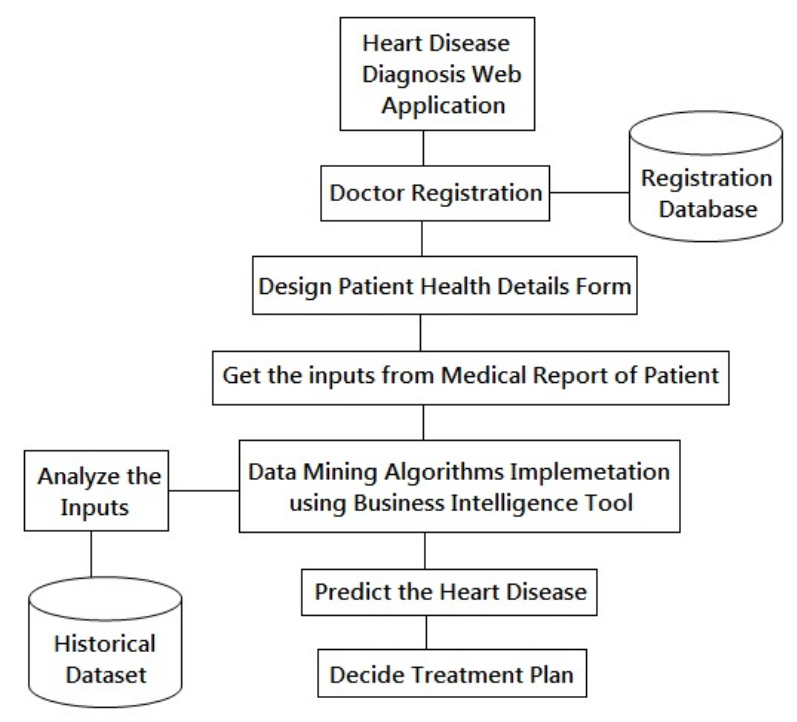

Fig. 4. System Architecture

\section{SYSTEM ARCHITECTURE}

In Heart disease diagnosis process, firstly health history of the patient is discussed. During that discussion, different symptoms data is collected with medical test report values.The symptoms data is very much necessary because correct diagnosis is only possible after understanding the exact reasons behind the heart disease. After collecting the symptoms data from patient, that data is analyzed using proper historical dataset. After analysis, the different single data mining prediction algorithms are used in diagnosis process and results are achieved. For accurate prediction of heart disease,the output of single data mining techniques are combined and compared with threshold value for diagnosis and accordingly treatment plan is decided.Then the diagnosis result of single data mining techniques and Hybrid data mining is compared and best treatment is recommended.

\section{HYBRID DATA MINING ALGORITHM}

Accurate diagnosis and treatment given to patients have been major issues emphasized in medical services. In recent times, research is ongoing for investigating data mining techniques to handle the error and complexity of treatment processes for healthcare service providers.

The concept of hybridization of data mining techniques is implemented using following algorithm:

(1) Let $M$ be a set of single data mining techniques, $\mathrm{M}=\{\mathrm{x} 1, \mathrm{x} 2, \mathrm{x} 3, \mathrm{x} 4, \mathrm{x} 5\}$ and $\mathrm{S}=$ Total support cases available in the dataset used to calculate the baseline accuracy or probability of Heart disease diagnosis and to decide treatment.

(2) Get the input attributes A1,A2,..,A13 from patient's Medical Report and Calculate the baseline accuracy or probability by applying single data mining techniques to diagnosis dataset $\mathrm{D}$, where $x \varepsilon M$.

The equation to find baseline accuracy or probability is:

$$
A=\frac{n(S)}{S} \times 100 \quad \text { Where, } S>n(S)
$$


Where, $A=$ Baseline Accuracy or probability, $\mathrm{n}(\mathrm{S})=$ Selected support cases Or Patient $\log$ $\mathrm{S}=$ Total support cases

(3) Calculate the output $\mathrm{O}$ of each single data mining technique which will come in binary values " 0 " and " 1 ". Where,

$\mathrm{O}=0$ (Zero) $=$ Patient does not exist heart disease.

$\mathrm{O}=1$ = Patient does exit heart disease.

(4) Calculate the baseline accuracy or probability by applying Hybrid $(\mathrm{H})$ data mining techniques to diagnosis dataset $\mathrm{D}$ using following equation :

$$
\mathrm{H}=(\mathrm{O} 1+\mathrm{O} 2+\mathrm{O} 3+\mathrm{O} 4+\mathrm{O} 5)
$$

Where,

$$
\begin{aligned}
& \text { O1 = Output of Naive Base algorithm } \\
& \text { O2 = Output of Decision Tree algorithm } \\
& \text { O3 = Output of Neural Network algorithm } \\
& \text { O4 = Output of Association Rule algorithm } \\
& \text { O5 = Output of Linear Regression algorithm }
\end{aligned}
$$

(5) Compare the output of Hybrid data mining techniques $\mathrm{H}$ with Threshold Value (3.0) output of Single data mining techniques M.

i) Check if $\mathrm{H}=(\mathrm{O} 1+\mathrm{O} 2+\mathrm{O} 3+\mathrm{O} 4+\mathrm{O} 5) \geq 3.0$

ii) If 'YES' goto step 6 and 8 , Else goto step 7 .

(6) Patient does exist heart disease.

(7) Patient does not exist heart disease.

(8) Suitable treatment/prescription for patient, operate it.

\section{PATIENT MEDICAL DATASET AND RESULT}

The dataset used in this study is the Cleveland Clinic Foundation Heart disease data set available at http://archive.ics.uciat.edu/ml/datasets/Heart+Disease.For experiment 13 attributes are considered:

Diagnosis(value 0: $\leq 50 \%$ diameter narrowing (no heart disease); (value $1: \geq 50 \%$ diameter narrowing (has heart disease)

Key attribute: PatientID (Patient's identification number)

(1) Sex (value 1: Male; value 0 : Female)

(2) Chest Pain Type (value 1: typical type 1 angina, value 2: typical type angina, value 3: non-angina pain; value 4: asymptomatic)

(3) Fasting Blood Sugar (value $1: \geq 120 \mathrm{mg} / \mathrm{dl}$; value $0: \leq 120$ $\mathrm{mg} / \mathrm{dl})$

(4) Restecg - resting electrographic results (value 0: normal; value 1: 1 having ST-T wave abnormality; value 2 : showing probable or definite left ventricular hypertrophy)

(5) Exang - exercise induced angina (value 1: yes; value 0: no)

(6) Slope - the slope of the peak exercise ST segment (value 1: unsloping; value 2: flat; value 3: downsloping)

(7) CA - number of major vessels colored by floursopy (value 0 3)

(8) Thal (value 3: normal; value 6: fixed defect; value 7: reversible defect)

(9) Trest Blood Pressure (mm Hg on admission to the hospital)

(10) Serum Cholesterol ( $\mathrm{mg} / \mathrm{dl})$

(11) Thalach - maximum heart rate achieved

(12) Oldpeak - ST depression induced by exercise relative to rest

(13) Age in Year
Table 1. Result Set for Heart Disease Probability and Prediction

\begin{tabular}{|l|c|c|c|c|}
\hline Type & Technique & Support Cases & Probability & Output \\
\hline Single & Naive Base & 285 & 96 & 1 \\
\hline Single & Decision Tree & 135 & 75 & 0 \\
\hline Single & Association Rule & 297 & 100 & 1 \\
\hline Single & Neural Network & 175 & 84.17 & 1 \\
\hline Single & Linear Regression & 297 & 99.57 & 1 \\
\hline Hybrid & Addition of o/p's $\geq 3.0$ & - & - & Disease exist \\
\hline
\end{tabular}

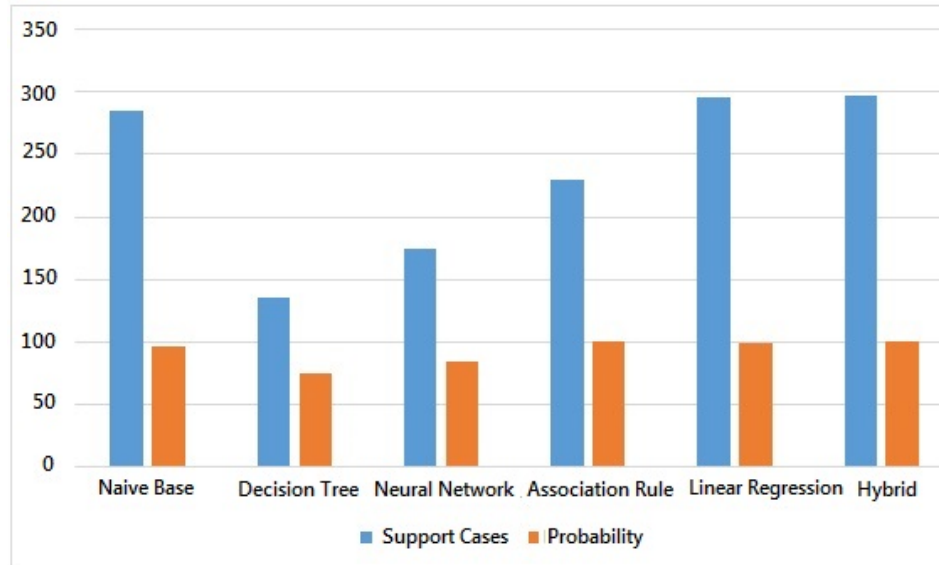

Fig. 5. Graphical Representation of Disease Probability and Prediction

\section{CONCLUSION}

A single mistake in diagnosis leads to incorrect treatment and ultimately patient's life would be in trouble.The "Trust" is very vital factor between doctor and patient.The different classification and prediction single data mining algorithms namely Naive Base,Decision Tree,Neural network ,Association Rule and Linear Regression are implemented.Each algorithm contains certain functions which are helpful to diagnose the heart disease.For perfect analysis of heart disease, the outputs of each algorithm is combined and compared with threshold value 3.0.Here combination of output is considered as "Hybridization". If the addition of that outputs are greater than 3.0 then the presence of heart disease is finalized and accordingly treatment is recommended.

Due to advance computer data mining techniques like hybrid data mining technique,doctors are quite relax to treat any patient and especially in case of heart disease,the hybrid technique carried drastic change.All algorithms showing diverse output,due to that it is difficult to conclude which one is better and perfect for diagnosis.The accuracy of every algorithm is calculated by analyzing historical dataset.The prediction of heart disease is done using binary values that is " 0 " and " 1 ". If the patient does exist heart disease then output shown by web application is " 1 " and if patient does not exist heart disease then web application shows the output " 0 ".

In Future,Additional data mining techniques can be incorporated to provide better results for better life of human being and the hybridization of data mining techniques will be useful in diagnosis and treatment plans of multiple diseases like-Cancer prediction, HIV prediction. 


\section{REFERENCES}

[1] Mai Shouman, Tim Turner, Rob Stocker,Using Data Mining Techniques in Heart Disease Diagnosis and Treatment. 978-1-4673-0484-9/12/ 2013 IEEE

[2] Obenshain, M.K., Application of Data Mining Techniques to Healthcare Data. Infection Control And Hospital Epidemiology, 2004.

[3] Rajkumar, A. and G.S. Reena, Diagnosis Of Heart Disease Using Data mining Algorithm. Global Journal of Computer Science and Technology, 2010. Vol. 10 (Issue 10).

[4] Razali, A.M. and S. Ali, Generating Treatment Plan in Medicine: A Data Mining Approach. American Journal of Applied Sciences, 2009. 6 (2): 345-351.

[5] Saad Ali, S.N., Developing treatment plan support in outpatient health care delivery with decision trees technique. Springer-Verlag Berlin Heidelberg, 2010. Part II, LNCS 6441, pp. $475 ? 482$.

[6] Kim, J., A Novel Data Mining Approach to the Identification of Effective Drugs or Combinations for Targeted Endpoints : Application to Chronic Heart Failure as a New Form of Evidence based Medicine. Cardiovascular Drugs and Therapy, Springer 2005. 18p. 483 ? 489.

[7] Srinivas, K., B.K. Rani, and A. Govrdhan, Applications of Data Mining Techniques in Healthcare and Prediction of Heart Attacks. International Journal on Computer Science and Engineering (IJCSE), 2010. Vol. 02, No. 02: p. 250-255.

[8] Das, R., I. Turkoglu, and A. Sengur, Effective diagnosis of heart disease through neural networks ensembles. Expert Systems with Applications, Elsevier, 2009. 36 (2009): p.7675?7680.

[9] Lee, I.-N., S.-C. Liao, and M. Embrechts, Data mining techniques applied to medical information. Med. inform, 2000.K. Elissa.

[10] Aswathy Wilson, Jismi Simon, Liya Thomas, Soniya Joseph, Data Mining Techniques For Heart Disease Prediction, Volume 3, No.2, February 2014, IJACST Journal

[11] Mai Shouman, Tim Turner, Rob Stocker,Using Decision Tree for diagnosing Heart Disease Patients, Proceedings of the 9-th Australasian Data Mining Conference (AusDM'11), Ballarat, Australia,2011.

[12] http://www.ecgguru.com

[13] G.Subbalakshmi, Decision Support in Heart Disease Prediction System using Naive Bayes, Indian Journal of Computer Science and Engineering(IJCSE)

[14] http://cs231n.github.io/neural-networks-1/

[15] https://msdn.microsoft.com/en-us/library/ms174824.aspx 